\title{
VELOCITY DISPERSIONS AND METALLICITIES OF BOX-SHAPED GALAXIES
}

\author{
R. E. DE SOUZA, S. DOS ANJOS, B. BARBUY \\ 1 IAG-USP, CP 9638, 01065-970 São Paulo, Brazil
}

\begin{abstract}
Velocity dispersions and $\mathrm{Mg}_{2}$ indices were measured from CCD spectra of $13 \mathrm{~S} 0$-box-shaped galaxies and 5 ellipticals. Evidence for a different relation between velocity dispersion and metallicity, relative to ellipticals, was found for our S0 sample; we attribute this difference to the S0 disk component, affecting both the mean velocity dispersion and mean metallicity.
\end{abstract}

\section{Introduction}

The objects used in this work were selected from the catalog of box-shaped galaxies (de Souza \& dos Anjos, 1987), classified from visual inspection of the ESO-B films and POSS prints. A sample constituted mainly of S0 edge-on galaxies, extracted from this list, was selected for photometric and spectroscopic observations. The photometric properties of $10 \mathrm{~S} 0$ box-shaped galaxies, 9 of which are in common with the present sample, is presented elsewhere by dos Anjos \& de Souza (1992). The present work is concerned to the analysis of spectroscopic medium resolution data for $13 \mathrm{~S} 0$ $\mathrm{Sa}$ and $5 \mathrm{E}$ galaxies, for which velocity dispersions and $\mathrm{Mg}_{2}$ indices were measured. The spectroscopic observations were done with the Cassegrain spectrograph at the 1.6m Boller \& Chivens telescope of the Laboratório Nacional de Astrofísica (LNA), Brazópolis, Brazil, in january/1991 and in september/1991. We use a grid of 900 $\mathrm{tr} / \mathrm{mm}$ centered at $510 \mathrm{~nm}$ with a reciprocal dispersion of $45.9 \AA / \mathrm{mm}$ corresponding to $1.009 \AA /$ pixel in the detector. The spectral coverage was $580 \AA$ and the slit aperture was $400 \mu$ corresponding to 4 arcsec projected in the sky and a spectral resolution of $4.1 \AA$, measured from the comparison spectrum of a HeAr lamp. The detector was a GEC CCD ( $400 \times 600$ pixels ) with $22 \mu /$ pixel. Each object was observed using three exposures of 20 minutes centered at the nucleus. The reductions were carried out with the VAX 8530 of the Astronomy Department of IAG/USP, using the eVe package routines developed at Meudon Observatory. The photometric calibration was done by observing standard spectrophotometric stars from the list of Stone and Baldwin (1983), and Taylor (1984). The extinction correction was estimated using broad band photoeletric photometry measurements observed at LNA during the period 89-91.

\section{Radial velocities, velocity dispersions and $\mathbf{M g}_{2}$ indices}

In the determination of velocity dispersion we basically adopt the Fourier Quotient algorithm described by Sargent et al. (1977), using as reference spectra a sample of KOIII giant stars. After the observation of each galaxy we have observed at least one reference star in the same night and in the same sky region of the program objects. The radial velocities of the reference stars were determined by direct identification of prominent isolated spectral features: $\mathrm{H} \beta(\lambda 4861.3), \mathrm{Fe}(\mathrm{lambda} 4910.03), \mathrm{Fe}(\lambda 4920.51), \mathrm{Fe}(\lambda 5079.75), \mathrm{Mg}(\lambda 5183.30)$, 
$\mathrm{Fe}(\lambda 5226.87), \mathrm{Fe}(\lambda 5328.05)$ and $\mathrm{Fe}(\lambda 5446.92)$. The mean square error in the final velocities of the stars is of the order of $10 \mathrm{~km} . \mathrm{s}^{-1}$.

The $\mathrm{Mg}_{2}$ index was measured from the calibrated spectra in the way described by Burstein et al. (1984), where the $\mathrm{Mg}_{2}$ bandpass is located at $\lambda \lambda 515.6-519.725 \mathrm{~nm}$ with blue continuum at $489.7-495.825 \mathrm{~nm}$ and red continuum at $530.3-536.675 \mathrm{~nm}$.

As pointed out by Terlevich and Davies (1981) there is a relation good correlation between velocity dispersion and mettalicity. We confirm the presence of this correlation for our SO sample as can be seen in Fig. 1. However the observed correlation is steeper than the one observed in the sample of pure ellipticals of Faber et al. (1989). The presence of this relation is probably related to differences in the evolution of galaxies of different masses (Binney, 1989). In low mass galaxies the gravitational potential is not suficcient to bound the chemically enriched galactic wind driven by supernovae explosions, and as a result they can loose as much as $70 \%$ of their original mass (Arimoto, Yoshi, 1987). On the contrary larger mass galaxies retain the enriched material increasing therefore their mettalicities. In Fig 1. we show the trend present in their model

\section{References}

dos Anjos, S., de Souza, R.E.: 1992, this Symposium

Arimoto, N., Yoshii, Y.: 1987, A\&A 173, 23

Binney, J., Tremaine, S.: 1987, Galactic Dynamics, Princeton Univ. Press

Burstein, D., Faber, S.M., Gaskell, C.M., Krumm, N.: 1984, ApJ, 287, 586

Faber, S.M., Wegner, G., Bustein, D., Davies, R.L., Dressler, A., Lynden-Bell, D.,

Terlevich, R.J.: 1989, ApJS, 69, 763

Terlevich, R., Davies, R.L., Faber, S.M., Burstein, D.: 1981, MNRAS 196, 381

Taylor, B.J.: 1984, ApJS 54, 259

Sargent, W.L.W., Schechter, P.L., Boksenberg, A., Shortridge, K.: 1977, ApJ 212, 326

de Souza, R.E., dos Anjos, S.: 1987, A\&AS 70, 465

Stone, P.S., Baldwin, J.A. : 1983, MNRAS 204, 347ex

Figure 1 - Velocity dispersions $\left(\mathrm{km} \mathrm{s}^{-1}\right)$ vs $[\mathrm{M} / \mathrm{H}]$

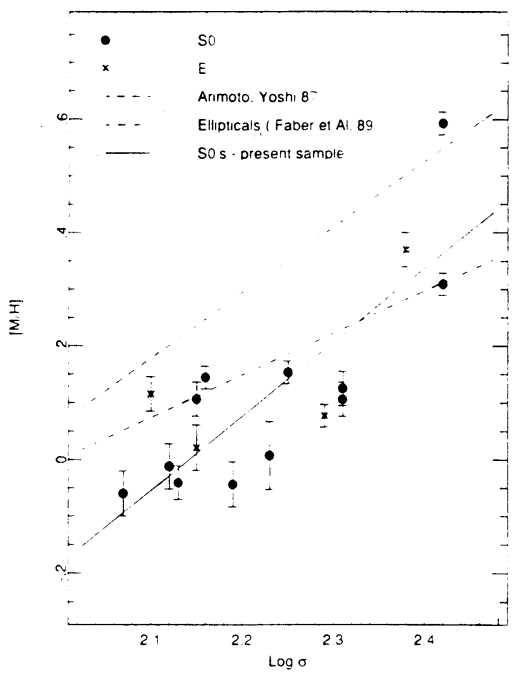

